\title{
THE DIFFERENTIAL EMISSION MEASURE OF $\lambda$ And
}

\author{
M.Landini ${ }^{1}$, B.C.Monsignori Fossi ${ }^{2}$ and R.Pallavicini ${ }^{2}$ \\ $1_{\text {(Department of Physics,University of Naples, Italy) }}$ \\ 2(Arcetri Astrophysical Observatory,Florence, Italy)
}

\begin{abstract}
Simultaneous X-ray and UV observations of $\lambda$ And from EXOSAT and IUE are analyzed and the differential emission measure as a function of temperature is derived.
\end{abstract}

\section{1 - INTRODUCTION}

$\lambda$ And is a well known single line spectroscopic binary with an orbital period of $20.5^{\mathrm{d}}$. Its very low mass function $\left(0.006 \mathrm{M}_{\odot}\right)$ and no evidence of eclipses indicate that the system is seen almost pole on. A photometric optical variability with a period of $54^{\mathrm{d}}$ has been shown to be produced by a system of two large spots on the primary star which, therefore, does not rotate synchronously with the orbital motion. The primary is a G 7-8 III-IV star which has long been known to have strong chromospheric activity in the Ca II (Gratton, 1950) and Mg II lines (Baliunas and Dupree, 1982). Its chromospheric and coronal activity is also confirmed by microwave (Bath and Wallestein,1976), X-ray (Walter and Bowyer,1980; Vaiana et al.,1981) and UV emissions( Linsky et al. 1978).These observations indicate that $\lambda$ And is a RS CVn star (Hall, 1981) and one of the brightest members of this class.

Observations of $\lambda$ And with the EXOSAT satellite were included in a large program devoted to the study of activity in late type stars ( Pallavicini et al., 1988 ). On November 11, 1985 simultaneous observations were obtained with EXOSAT LE and ME experiments and with IUE. For the purpose of the present analysis, the ME data have been binned in 3 bands (table 1 ).

Table 1

\begin{tabular}{|c|c|c|c|c|c|c|c|c|}
\hline \multirow{3}{*}{$\begin{array}{c}\text { EXOSAT ME } \\
(\mathrm{keV}) \\
1.17-2.14\end{array}$} & \multicolumn{6}{|c|}{$\begin{array}{c}\text { Observations of } \lambda \text { And on Nov } 11,1985 \\
\text { EXOSAT LE }\end{array}$} & \multicolumn{2}{|l|}{ IUE } \\
\hline & \multicolumn{2}{|c|}{$\mathrm{c} \mathrm{s}^{-1}$} & \multicolumn{3}{|c|}{$\mathrm{cs}^{-1}$} & & \multicolumn{2}{|c|}{$\mathrm{c} \mathrm{s}^{-1}$} \\
\hline & 7.54 & $10^{-1}$ & 3 Lex & 4.815 & $10^{-1}$ & N V & 2.5 & $10^{-2}$ \\
\hline $2.14-3.40$ & 8.58 & $10^{-1}$ & 4 lex & 3.957 & $10^{-} 1$ & C II & 1.23 & $10^{-1}$ \\
\hline $3.40-5.26$ & 4.30 & $10^{-1}$ & $\mathrm{Al} / \mathrm{Pa}$ & 1.279 & $10^{-1}$ & Si IV & 1.1 & $10^{-1}$ \\
\hline & & & Boron & 3.253 & $10^{-2}$ & C IV & 3.11 & $10^{-1}$ \\
\hline
\end{tabular}

The aim of this paper is to analyze these observations in order to deduce the differential emission measure as a function of temperature. Uncertainties are assumed to be $\pm 10 \%$ for the EXOSAT data and $\pm 30 \%$ for the IUE data.

\section{2 - THE DIFFERENTIAL EMISSION MEASURE DISTRIBUTION.}

It is not possible to reproduce both the UV and X-ray observations with a single temperature model; this is well known in the solar case and is clearly shown by fig. 1, where for each temperature we plot the emission measure that would be needed to reproduce the observed emissions. The same figure suggests that most of the emission in the ME and LE bands must come from a " quasi isothermal " plasma with a temperature higher than $2010^{6} \mathrm{~K}$, while no isothermal solution appears to be possible for the UV lines. 


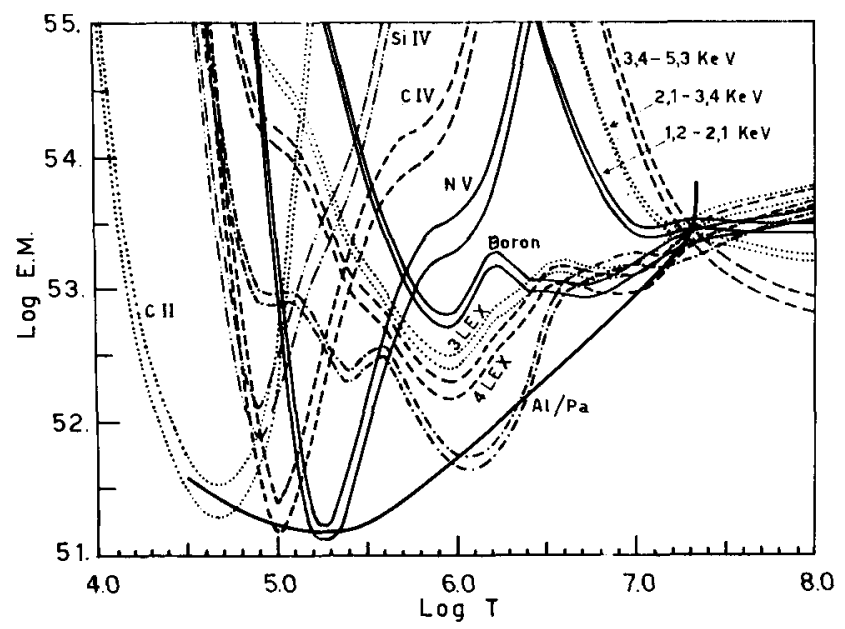

FIG. 1 - Log of the emission measure versus temperature allowed by the observed fluxes (including errors) in binned $M E$ bands (1.17-2.14 KeV, 2.14-3.4 KeV, 3.4-5.26 KeV), LE filters (Boron, 3Lexan, 4 Lexan, AllPa) and UV lines (C II, C N, NV, Si IV).

The envelope of curves shown in fig. 1 suggests the shape of the differential emission measure (d.e.m.) as a function of the temperature. We assume the following "parameterized" form of d.e.m.:

$$
\mathrm{n}^{2}(\mathrm{dV} / \mathrm{dT})=\mathrm{Ce}^{\mathrm{T}} \mathrm{o} / \mathrm{T}\left(\mathrm{T} / \mathrm{T}_{\mathrm{c}}\right)^{\beta}\left[1-\left(\mathrm{T} / \mathrm{T}_{\mathrm{c}}\right)^{\gamma}\right]^{-1 / 2}
$$

Apart from the exponential term, which modulates the low temperature region ( $\mathrm{UV}$ emission), this form is suggested by a theoretical investigation of the energy balance (Landini and Monsignori Fossi, 1975,1981 ) and has been proved to be useful also for solar active regions (Monsignori Fossi and Landini, 1988) .

The plasma emissivity for each spectral region of interest has been integrated over the assumed d.e.m. and a best $\chi^{2}$ fit procedure has been used to evaluate the free parameters $T_{0}, T_{c}, b, g$ and $C$; in the present case the best fit procedure has been applied to all data in table 1 except the $\mathrm{C}$ II line which may be optically think and the $\mathrm{Al} / \mathrm{Pa}$ filter, which proved to give an unusually low flux (see later).

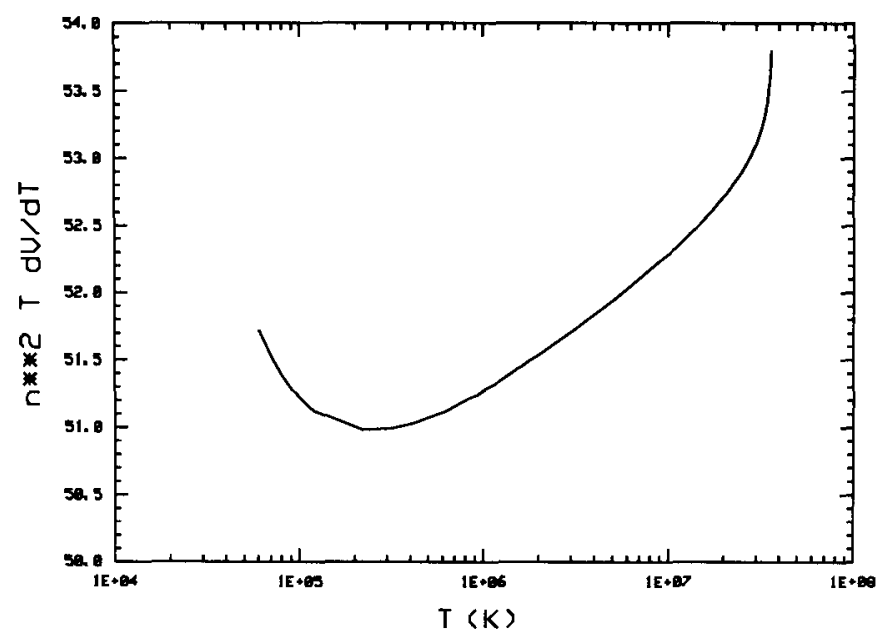

FIG. 2 - The differential emission measure distribution that allows the best $\chi^{2}$ fit between predicted and observed data. 
The following values give a reasonable good fit ( reduced $\chi^{2}=1.2$ ): $\log T_{c}=7.56 ; \log T_{0}=5.4 ; \gamma=.64$; $\beta=.015 ; \mathrm{C}=1.510^{45} \mathrm{~cm}^{-3} \mathrm{~K}^{-1}$.

The differential emission measure is plotted in fig.2 and is used to generate a synthetic spectrum (Landini and Monsignori Fossi, 1984) of the radiation emitted by the portion of the loop at temperatures larger than $610^{4} \mathrm{~K}$.

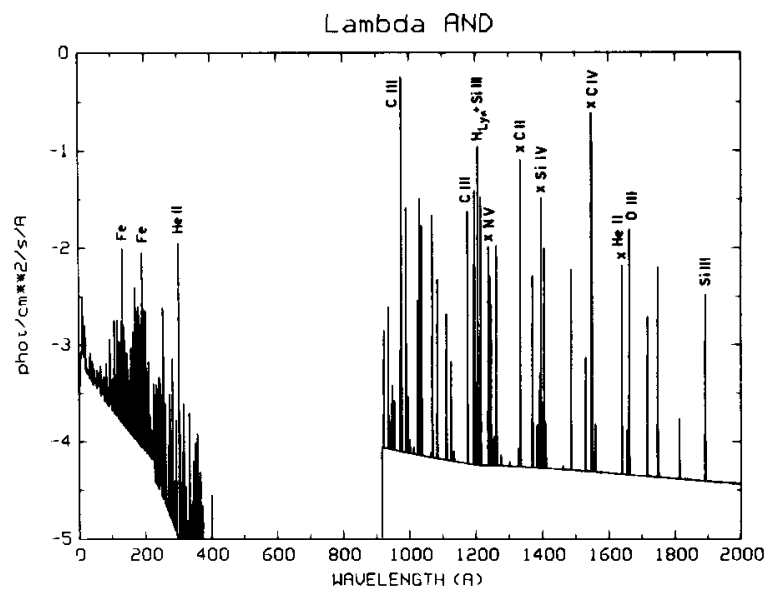

FIG. 3 - The synthetic spectrum from 1 to $2000 \mathrm{~A}$ obtained for $\lambda$ And assuming the parameterized differential emission measure distribution shown in fig.2. The interstellar absorption, evaluated with a column density $N_{H}=5.210^{18} \mathrm{~cm}^{-2}$, affects the region from 200 to $980 \mathrm{~A}$.

The spectral distribution over the region 1-2000 A is shown in fig. 3: the most prominent spectral lines are indicated and crosses give the observed fluxes for the IUE lines. A few lines which have not been used in the $\chi^{2}$ fit are present in the synthetic spectrum and need comments: Si IV (1397 A) is too low by a factor 3 than predicted: this fact was already noticed and is attributed to the density dependence of ion abundance (Jordan et al., 1987). He II $(1640 \mathrm{~A})$ in $\lambda$ And is much higher than predicted: this fact can be attributed to an overpopulation of the upper level due to recombination of He nuclei following photoionization by photons with $\lambda<227 \mathrm{~A}$. These photons are much more numerous in $\lambda$ And than in the Sun, due to the higher coronal temperature of this star (Linsky et al., 1978). Photoionization modifies the ionization balance of the He ions and this fact may also explain the disagreement of the $\mathrm{Al} / \mathrm{Pa}$ signal which is be affected by the strong He II $303 \mathrm{~A}$ line visible in the synthetic spectrum. Also a sligthly different value for the interstellar absorption may change the expected $\mathrm{Al} / \mathrm{Pa}$ signal.

In the Sun, transition region and coronal emission originates from loop-like structures which to first approximation can be considered of costant pressure and costant cross section. We can make the same assumption for $\lambda$ And. Two large spots, covering $12 \%$ of the surface of the primary star was inferred by Bopp and Noha (1980), at about $30^{\circ}$ in latitude and separated by $160^{\circ}$ in longitudine. If we assume that they indicate the presence of two "preferential longitudines" of activity we may postulate the existence of a large loop connecting them.

For a star radius is 5-6 $\mathrm{R}_{\Theta}$, the two spots are about $10^{12} \mathrm{~cm}$ apart and this value can also be assumed as a reasonable estimate of the half length $\mathrm{L}$ of the loop. If this loop is similar to solar ones, an aspect ratio of 0.1 may be assumed and the loop cross-section is given by:

$$
S=\pi 10^{-2} \mathrm{~L}^{2} \simeq 310^{22} \mathrm{~cm}^{2}
$$

Since the projected disk area of the star is about $4.510^{23} \mathrm{~cm}^{2}$, the loop cros-section is somewhat less than $10 \%$ of the stellar disk, very near the value obtained from the optical observations. 
Assuming a constant pressure and a constant cross-section, the semilength of the loop is given by:

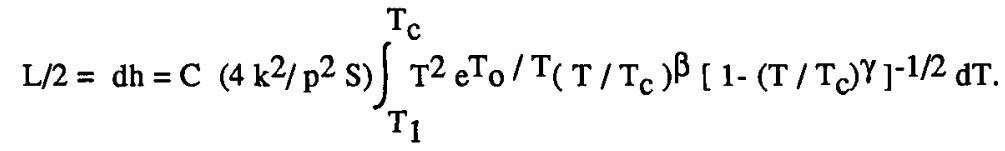

With $\mathrm{T}_{1}=210^{4} \mathrm{OK}$ and the best-fit parameters given above, one derives $\mathrm{p}=15 \mathrm{dyn}^{-2}$, using the mentioned values of $S$ and $L$.

\section{CONCLUSIONS}

The analysis of X-ray and UV emission of $\lambda$ And observed with EXOSAT (ME and LE) and IUE shows that:

- a continous distribution of d.e.m. with temperature up to a maximum temperature $\simeq 3510^{6} \mathrm{o} \mathrm{K}$ can reproduce (reduced $\chi^{2} \simeq 1.2$ ) all available observations. The worst fit occurs for the low energy part of the ME spectrum, which requires a somewhat larger d.e.m. around $510^{6} \mathrm{o}_{\mathrm{K}}$, and for the $\mathrm{Al} / \mathrm{Pa}$ filter, which is lower than theoretical predictions (a larger interstellar column density ?);

- the d.e.m. [ $\left.\mathrm{T} \mathrm{n}^{2}(\mathrm{dV} / \mathrm{dT})\right]$ presents a minimum around $210^{5} \mathrm{o}_{\mathrm{K}}$ and increases at higher temperatures; - the derived d.e.m. is compatible with a loop model having: half length $=10^{12} \mathrm{~cm}$, cross-section $=3$ $10^{22} \mathrm{~cm}^{2}$, and pressure $=15 \mathrm{dyn} \mathrm{cm}^{-2}$. In contrast to the model of Rosner et al.(1978) the heating deposition along this loop turns out to be non uniform.

\section{REFERENCES}

Baliunas S.L. and Dupree A.K.: 1982, Ap.J. 252, 668

Bath G.T. and Wallestein G., Publ.Astr.Soc.Pac.:1976, 88, 759

Bopp B.W. and Noha P.V., Publ.Soc.Pac.:1980, 92,717,

Gratton L.:1950, Ap.J., 111, 31

Hall D.S. : 1981, in 'Solar Phenomena in Star and Stellar Systems' R. Bonnet and A. Dupree eds.,p.431

Jordan C. et al.:1987,Mon.Not.R.astr.Soc.,225,903

Landini M. and Monsignori Fossi B.C.:1975, Astron. Astrophys.,42,213

Landini M. and Monsignori Fossi B.C.:1981, Astron. Astrophys.,102,391

Landini M. and Monsignori Fossi B.C.:1984, Physica Scripta,T7,53

Linsky J.L. et al.,Nature : $1978,275,389$

Monsignori Fossi B.C. and Landini M.:1988 in "Activity in Cool Star Envelopes" O.Havens et al. eds.,237

Pallavicini R., Monsignori Fossi B.C., Landini M. and Schmitt J.H.M.M. : 1988, A.Ap. 191, 109

Rosner R.,Tucker W.H. and Vaiana G.S.,1978, Ap.J., 220,643

Vaiana G.S.et al.: 1981, Ap.J.,245,163

Walter F.and Bowyer S.:,1981, Ap.J.245, 671 